\title{
Long-term survival in patients with primary intracranial germ cell tumors treated with surgery, platinum-based chemotherapy, and radiotherapy: a single-institution study
}

\author{
Hiroyuki Shimizu, MD, Kazuya Motomura, MD, PhD, Fumiharu Ohka, MD, PhD, \\ Kosuke Aoki, MD, PhD, Kuniaki Tanahashi, MD, PhD, Masaki Hirano, MD, PhD, \\ Lushun Chalise, MD, PhD, Tomohide Nishikawa, MD, Junya Yamaguchi, MD, \\ Jun Yoshida, MD, PhD, Atsushi Natsume, MD, PhD, and Toshihiko Wakabayashi, MD, PhD \\ Department of Neurosurgery, Nagoya University School of Medicine, Nagoya, Japan
}

\begin{abstract}
OBJECTIVE The current study aimed to evaluate the treatment outcomes and toxicities of patients with intracranial germ cell tumors (GCTs).

METHODS This study retrospectively included 110 consecutive patients (70 patients in the germinomatous group and 40 patients in the nongerminomatous GCT [NGGCT] groups) receiving surgery, platinum-based chemotherapy, and radiotherapy for newly diagnosed primary intracranial GCTs. In the authors' protocol, patients with GCTs were further divided into the following four groups: the germinomatous group and the NGGCT groups (mature teratoma, intermediate prognosis, or poor prognosis).

RESULTS The median overall survival (OS) and progression-free survival (PFS) rates of the patients in the germinomatous group were significantly higher than those in the NGGCT group $(p<0.001)$. The $5-, 10-$, and 20 -year OS rates in the germinomatous group were $97.1 \%, 95.7 \%$, and $93.2 \%$, respectively, with a median follow-up of 11.0 years. On the contrary, the 5-, 10-, and 20-year OS rates in the NGGCT group were $67.3 \%, 63.4 \%$, and $55.4 \%$, respectively. The 5-, 10 -, and 20-year PFS rates were $91.4 \%, 86.6 \%$, and $86.6 \%$, respectively, in the germinomatous group, whereas those of the NGGCT group were approximately $67.4 \%, 60.2 \%$, and $53.5 \%$, respectively. Based on the four types of classification in our study, the 5-, 10-, and 20-year OS rates in the NGGCT intermediate prognosis group were $78.9 \%, 71.8 \%$, and $53.8 \%$, respectively. On the contrary, the 3- and 5-year OS rates in the NGGCT poor prognosis group were $42.9 \%$ and $34.3 \%$, respectively. Moreover, toxicities with the treatment of intracranial GCTs were found to be tolerable in the present study population. The multivariate survival models for OS in the NGGCT intermediate prognosis and poor prognosis groups demonstrated that only the alpha-fetoprotein status was significantly associated with worsened OS (HR 3.88, $95 \% \mathrm{Cl} 1.29-11.66 ; p=0.02$ ).

CONCLUSIONS The authors found that platinum-based chemotherapy and radiotherapy result in favorable survival outcomes in patients with germinomatous GCTs. Clinical outcomes were still unfavorable in the NGGCT intermediate prognosis and poor prognosis groups; therefore, a new protocol that increases the survival rate of patients belonging in both groups should be considered.
\end{abstract}

https://thejns.org/doi/abs/10.3171/2020.6.JNS20638

KEYWORDS intracranial germ cell tumor; platinum-based chemotherapy; radiotherapy; germinomatous; nongerminomatous; oncology

$\mathrm{I}$ NTRACRANIAL germ cell tumors (GCTs) account for $2.2 \%$ of all primary brain tumors in Japan. ${ }^{1}$ This occurrence rate is more frequently reported in individuals living in Asian countries compared with those living in
Western countries such as the United States $(0.4 \%){ }^{2}$ The majority of patients with GCTs are younger than 20 years, and approximately $55 \%$ of these patients are between 10 and 19 years of age at diagnosis. ${ }^{1}$ Men are more commonly

ABBREVIATIONS AFP = alpha-fetoprotein; $\beta-h C G=$ beta-human chorionic gonadotropin; $C B D C A+V P-16=$ carboplatin and etoposide; $C D D P+V P-16=$ cisplatin and etoposide; GCT = germ cell tumor; GTR = gross-total resection; ICE = carboplatin, etoposide, and ifosfamide; NGGCT = nongerminomatous GCT; OS = overall survival; PFS = progression-free survival; STGC = syncytiotrophoblastic giant cell; TICS-J = Japanese version of the Telephone Interview for Cognitive Status.

SUBMITTED February 28, 2020. ACCEPTED June 1, 2020.

INCLUDE WHEN CITING Published online October 2, 2020; DOI: 10.3171/2020.6.JNS20638. 
affected than women, with incidence ratios of 4.5:1 in Japan $^{1}$ and 3.9:1 in the United States. ${ }^{3}$ Intracranial GCTs are most commonly observed in the pineal and suprasellar regions and basal ganglia, frequently presenting with bifocal and disseminated lesions. ${ }^{1}$

GCTs can be globally classified into the germinomatous group and the nongerminomatous GCT (NGGCT) group according to their histological features based on the WHO classification., ${ }^{4,5}$ Particularly, pure germinomas and germinomas with syncytiotrophoblastic giant cells (STGCs) comprise approximately $70 \%$ of all intracranial GCTs. ${ }^{1}$ NGGCTs account for approximately one-third of GCTs and include several histologies with different prognoses, including choriocarcinomas, yolk sac tumors, embryonal carcinomas, mature teratoma, immature teratoma, teratoma with malignant transformation, and mixed tumors. ${ }^{6-8}$

Considering that both types of GCTs are sensitive to radiation and chemotherapy, chemoradiotherapy plays a central role in the treatment for these tumors. Several clinical studies have shown promising results of combined chemoradiotherapies for the germinomatous type.9-12 Chemotherapy prior to radiation therapy is commonly applied for germinoma. The SIOP-CNS-GCT-96 trial revealed that chemotherapy using carboplatin and etoposide alternating with etoposide and ifosfamide, followed by reduced volume and dose radiation therapy, produced comparable outcomes to craniospinal irradiation alone. ${ }^{13}$ On the contrary, one group reported effective protocols using carboplatin or cisplatin with etoposide, followed by local radiotherapy of $30 \mathrm{~Gy}$ and additional maintenance chemotherapy in the treatment of NGGCTs. ${ }^{9}$ By analyzing 153 cases of histologically verified intracranial GCTs, one group reported that the 10- and 20-year overall survival (OS) rates of patients with pure germinomas were 93\% and $81 \%$, respectively. ${ }^{12}$ Another study evaluating the clinical outcomes of 405 germinomas reported that the 20and 30-year OS rates in the germinomatous group were $84.1 \%$ and $61.9 \%$, respectively.$^{14}$ On the contrary, patients with NGGCTs have relatively more unfavorable outcomes than those with germinomatous tumors, regardless of the involvement of more intensive therapies, including the resection of residual tumor after chemotherapy and highdose craniospinal irradiation. ${ }^{15,16}$ It has been reported that the 5-year OS rate of patients with NGGCTs is lower, ranging from $30 \%$ to $70 \%, 7,15,17$ than the OS rate for those with germinomatous tumors. The most effective treatment for the germinomatous group seems to be radiation therapy with neoadjuvant chemotherapy; however, a standard therapy for patients with NGGCTs has yet to be established. Since primary intracranial NGGCTs are considered to be significantly rare neoplasms with heterogeneous malignancies, conducting large prospective trials to evaluate the clinical outcomes of NGGCTs might be difficult.

In the present study, we performed a retrospective review of 110 consecutive patients receiving surgery, platinum-based chemotherapy, and radiotherapy for newly diagnosed primary intracranial GCTs, including 40 patients with NGGCTs. To identify an optimal GCT treatment strategy, we evaluated the treatment outcomes and toxicities of patients with GCTs.

\section{Methods \\ Patient Population}

We retrospectively analyzed 110 consecutive patients with newly diagnosed intracranial GCTs at Nagoya University Hospital (Nagoya, Japan) between August 1978 and March 2014. Information regarding age, sex, histological type, tumor location and extent, tumor markers such as alpha-fetoprotein (AFP) and beta-human chorionic gonadotropin $(\beta-\mathrm{hCG})$, radiological findings, chemoradiotherapy, and treatment protocol and outcomes was obtained from the patients' medical records. The study protocol was approved by the ethics committee of the Nagoya University Hospital in accordance with all provisions of the Declaration of Helsinki.

\section{Diagnosis of Intracranial GCTs}

The diagnosis of GCTs was based on histological findings, serum and CSF levels of AFP and $\beta$-hCG, and radiological features such as MRI findings. Among the 110 patients, 70 were histologically diagnosed with GCT established from tissue samples obtained via tumor resections or needle biopsies, while 40 were clinically diagnosed with GCT based on examinations and neuroimaging findings. The histological diagnosis of brain tumor was established in accordance with the 2007 or 2016 WHO classification. ${ }^{4,5}$ The patients were treated according to the institutional protocol of the Nagoya University School of Medicine. In this protocol, patients with GCTs are divided into the following four groups: the germinomatous group and the NGGCT groups-mature teratoma, intermediate prognosis, and poor prognosis. NGGCTs are often considered in a single tumor category because of their rarity. However, different patterns of recurrence and survival have been reported among different subtypes., ${ }^{9,12}$ Moreover, patients with NGGCTs have heterogeneous histological types, with a significant difference in disease prognosis. ${ }^{9}, 12,18$ Therefore, the NGGCT group is further divided into the following three groups: the mature teratoma group, NGGCT intermediate prognosis group, and NGGCT poor prognosis group.

Patients with pure germinomas and germinomas with STGCs were assigned to the germinomatous group. These tumors were diagnosed when there was a histopathological confirmation and/or slight elevations of serum or CSF $\beta$-hCG (5.0-50 IU/L) level with the absence of an elevated AFP level. If the elevated $\beta$-hCG level was detected in the blood or CSF, a biopsy was not necessarily performed to establish the clinical diagnosis. In patients without positive tumor marker results, a biopsy was performed to establish the definitive diagnosis based on histological confirmation. Patients in the germinomatous group who did not undergo biopsy were diagnosed with germinomas based on their elevated $\beta$-hCG levels. In the germinomatous group, even after the completion of induction chemotherapy, patients with residual tumor and/or persistently elevated serum or CSF $\beta$-hCG levels underwent second-look surgery.

On the contrary, all of the tumors in the NGGCT group were histologically verified by surgical biopsy. The NGGCT group includes mature teratomas, the intermediate prognosis group (immature teratomas, teratomas with 
malignant transformation, and mixed tumors mainly comprising germinomas or teratomas), and the poor prognosis group (choriocarcinomas, yolk sac tumors, embryonal carcinomas, and mixed tumors mainly comprising choriocarcinomas, yolk sac tumors, or embryonal carcinomas). The $\beta$-hCG level is markedly elevated in choriocarcinomas, often higher than $1000 \mathrm{IU} / \mathrm{L}$. Furthermore, $\beta$-hCG levels may be elevated in some patients with embryonal carcinomas and immature teratoma. The diagnosis of yolk sac tumors was based on a high AFP (> $2000 \mathrm{ng} / \mathrm{mL}$ ) level. The AFP level is also elevated in some embryonal carcinomas and immature teratoma.

\section{Treatment Protocol}

From 1978 to 2014, patients in the germinomatous group received 2 courses of chemotherapy comprising carboplatin $300 \mathrm{mg} / \mathrm{m}^{2}$ on day 1 and etoposide $100 \mathrm{mg} / \mathrm{m}^{2}$ on days 1-3 (CBDCA+VP-16 regimen), followed by radiation therapy $(30 \mathrm{~Gy})$ to the entire ventricle. Thereafter, one cycle of maintenance chemotherapy was administered. If there were remnant tumors after the first radiotherapy session, we added 10 Gy to the tumor bed. For patients with basal ganglia tumors, whole-brain radiotherapy (30 Gy) was selected. Patients with multifocal or disseminated lesions in this group received chemotherapy comprising carboplatin $300 \mathrm{mg} / \mathrm{m}^{2}$ on day 1 , etoposide $100 \mathrm{mg} / \mathrm{m}^{2}$ on days $1-3$, and ifosfamide $1500 \mathrm{mg} / \mathrm{m}^{2}$ on days $1-3$ (ICE regimen), followed by radiation therapy (30 Gy) to the whole brain and spine. Regarding radiotherapy, patients in the germinoma group planned to receive whole-ventricle or whole-brain radiotherapy or radiotherapy to the whole brain and spine. However, between 2000 and 2001, radiation therapy was omitted, and the treatment protocol in this period was chemotherapy alone.

In the NGGCT group, patients with mature teratomas were treated with resection alone. Patients in the NGGCT intermediate prognosis group received 1 course of CBDCA+VP-16 chemotherapy, followed by resection, and 30 Gy of radiotherapy to the whole ventricle. Subsequently, 2-6 courses of maintenance chemotherapy were administered. Salvage surgery should be considered after or during chemoradiotherapy if complete remission is not achieved. Patients in the NGGCT poor prognosis group received 2 courses of ICE chemotherapy and salvage surgery followed by radiation therapy (30 Gy) to the whole brain and spine. Maintenance chemotherapy for this group consisted of 4-6 courses of ICE chemotherapy.

From 1987 to 2000, some patients in both groups received 2 courses of chemotherapy comprising cisplatin $20 \mathrm{mg} / \mathrm{m}^{2}$ and etoposide $60 \mathrm{mg} / \mathrm{m}^{2}$ on days $1-5$ (CDDP+VP-16 regimen), followed by large focal radiation therapy (40 Gy). ${ }^{19}$ Subsequently, one cycle of maintenance chemotherapy was administered.

\section{Neuropsychological Assessment}

Long-term intracranial GCT survivors who received radiation therapy to the brain were assessed for neurocognitive impairment using the Japanese version of the Telephone Interview for Cognitive Status (TICS-J). ${ }^{20}$ The TICS-J survey is a concise, standardized examination that assesses cognitive functioning and is designed to be administered over the telephone. The TICS-J comprises 11 test items and is scored from 0 to 41 , with higher scores indicating better cognitive functioning. Patients scoring 33 or higher are considered to be nonimpaired. For patients who agreed to participate, the TICS-J questionnaire was administered in a standardized manner by one of our investigators (H.S.) to objectively assess the global cognitive status of the patients.

\section{Statistical Analyses in the Germinomatous Group}

All data were analyzed using IBM SPSS (version 26.0, IBM Corp.) for Windows (Microsoft Corp.). The median follow-up was assessed from the date of the first visit to the date of death or last follow-up. The OS and progressionfree survival (PFS) rates were estimated according to the Kaplan-Meier method, and survival curves were compared using the log-rank test. OS was calculated from the date of the first visit to the date of death or last follow-up. PFS was calculated from the date of the first visit to the date of the first evidence of tumor progression or recurrence, death, or last follow-up. Univariate and multivariate analyses were performed to determine the potential influence of baseline characteristics on survival. The effect of predictors on OS in the NGGCT intermediate prognosis and poor prognosis groups was investigated using the Cox proportional hazards model, adjusting for the major clinical prognostic factors, including age at diagnosis $(\leq 20$ years vs $>20$ years), sex (male vs female), tumor location (pineal regions vs other regions), AFP levels $(<300 \mathrm{ng} / \mathrm{mL}$ vs $\geq$ $300 \mathrm{ng} / \mathrm{mL}$ ), $\beta$-hCG levels (< $300 \mathrm{IU} / \mathrm{L}$ vs $\geq 300 \mathrm{IU} / \mathrm{L}$ ), extent of tumor resection (gross-total resection [GTR] vs non-GTR), multiplicity (absent vs present), and dissemination (absent vs present). Factors with no significant association with survival, at $\mathrm{p}>0.05$ in the univariate analysis, were eliminated. The remaining factors in the multivariate proportional hazards model $(\mathrm{p}<0.05)$ were considered to be independent predictors of survival in the NGGCT intermediate prognosis and poor prognosis groups.

\section{Results \\ Patient Characteristics}

Between August 1978 and March 2014, 110 consecutive patients (90 male and 20 female) with newly diagnosed intracranial GCTs were registered in this study. The median follow-up time was 11.0 years (range 0.5-37.8 years). Based on the patients' clinical characteristics summarized in Table 1, the median age at diagnosis was 14 years (range 1-63 years). Figure 1 shows the age distribution of the overall cohort diagnosed with intracranial GCTs in 5-year increments of diagnosis between 1978 and 2014. Based on the histological findings, serum/CSF levels of AFP and $\beta$-hCG, and radiological features, this study included patients with intracranial GCTs, including 40 pure germinomas 30 germinomas with STGC, 7 mature teratomas, 14 immature teratomas, 14 mixed GCTs, 4 yolk sac tumors, and 1 embryonal carcinoma. The majority of the tumors were located in the pineal region $(n=52$, $47.3 \%)$, followed by the suprasellar region $(n=39,35.5 \%)$ and the basal ganglia $(n=16,14.5 \%)$. 
TABLE 1. Clinical characteristics of 110 patients with GCTs

\begin{tabular}{|c|c|}
\hline Parameter & Value \\
\hline No. of patients & 110 \\
\hline \multicolumn{2}{|l|}{ Age, yrs } \\
\hline Median & 14 \\
\hline Range & $1-63$ \\
\hline \multicolumn{2}{|l|}{ Sex } \\
\hline Male & $90(81.8)$ \\
\hline Female & $20(18.2)$ \\
\hline \multicolumn{2}{|l|}{ Tumor type } \\
\hline Pure germinoma & $40(36.4)$ \\
\hline Germinoma w/ STGC & $30(27.3)$ \\
\hline Mature teratoma & $7(6.4)$ \\
\hline Immature teratoma & $14(12.7)$ \\
\hline Mixed GCT & $14(12.7)$ \\
\hline Yolk sac tumor & $4(3.6)$ \\
\hline Embryonal carcinoma & $1(0.9)$ \\
\hline \multicolumn{2}{|l|}{ Tumor location } \\
\hline Pineal & $52(47.3)$ \\
\hline Suprasellar & $39(35.5)$ \\
\hline Basal ganglia & $16(14.5)$ \\
\hline 4th ventricle & $2(1.8)$ \\
\hline Thalamus & $2(1.8)$ \\
\hline Cervical & $2(1.8)$ \\
\hline Frontal & $2(1.8)$ \\
\hline Occipital & $1(0.9)$ \\
\hline Cerebellum & $1(0.9)$ \\
\hline
\end{tabular}

Values represent the number of patients (\%) unless stated otherwise.

\section{Treatment for Intracranial GCTs Based on the Four Groups}

In the germinomatous group, 30 of the 70 patients were histologically diagnosed with pure germinomas with STGC. Forty patients were clinically diagnosed as being in the germinomatous group by slight elevations of serum or CSF $\beta$-hCG without elevated AFP (Table 2). Among the 70 patients, $47(67.1 \%)$ were treated with CBDCA+VP-16 chemotherapy, and $6(8.6 \%)$ received ICE therapy. Before 2000, 15 patients $(21.4 \%)$ were treated with CDDP+VP-16 chemotherapy. Two patients who did not receive chemotherapy were treated with only radiation therapy. The germinomatous group included $21 \mathrm{pa}-$ tients treated with radiotherapy to the whole ventricle, 18 patients with radiotherapy to the whole brain, 12 patients with radiotherapy to the craniospinal axis, and 16 patients with large focal radiation therapy. Three patients who did not receive radiotherapy were only treated with chemotherapy, including CBDCA+VP-16 or CDDP+VP-16 chemotherapy.

A total of 40 patients were assigned in the NGGCT group (Table 2). Seven patients with mature teratomas were treated with tumor resection without chemoradiotherapy. Patients in the NGGCT group were further divided into the following two groups: 19 patients in the intermediate prognosis group and 14 patients in the poor prognosis group. All 19 patients in the NGGCT intermediate prognosis group underwent partial, subtotal, or total tumor resection as the salvage surgery after a couple of rounds of chemotherapy. Thereafter, they received radiotherapy followed by 2-6 courses of chemotherapy. They received radiation therapy in various fields, such as the whole ventricle, whole brain, and craniospinal axis, and large focal radiation therapy, and also received chemotherapy, including CBDCA+VP-16, CDDP+VP-16, or ICE chemotherapy (Table 2). In the NGGCT poor prognosis group, all patients underwent salvage surgery after receiving 2 courses of chemotherapy. Moreover, 11 of the 14 patients $(78.6 \%)$ received ICE chemotherapy, and 5 of the 14 patients (35.7\%) were treated with radiotherapy to the craniospinal axis.

\section{Survival Data of Patients in the Germinomatous and NGGCT Groups}

The Kaplan-Meier estimates for OS and PFS in the germinomatous and NGGCT groups are shown in Fig. 2. The median OS and PFS rates of patients in the germinomatous group (not reached) were significantly higher than those in the NGGCT group (not reached) $(\mathrm{p}<0.001)$. The $5-, 10-$, and 20-year OS rates in the germinomatous group were $97.1 \%, 95.7 \%$, and $93.2 \%$, respectively, with a median follow-up of 11.0 years. On the contrary, the 5-, 10-, and 20-year OS rates in the NGGCT group were 67.3\%, $63.4 \%$, and $55.4 \%$, respectively. The 5-, $10-$, and 20 -year PFS rates were $91.4 \%, 86.6 \%$, and $86.6 \%$, respectively, in the germinomatous group, whereas those of the NGGCT group were approximately $67.4 \%, 60.2 \%$, and $53.5 \%$, respectively.

The NGGCT poor prognosis group demonstrated significantly worse OS and PFS rates compared with those of the germinomatous group and the NGGCT intermediate prognosis group ( $\mathrm{p}<0.001$ ) (Fig. 2). The 5-, 10-, and 20 -year OS rates in the NGGCT intermediate prognosis group were $78.9 \%, 71.8 \%$, and $53.8 \%$, respectively. On the contrary, the 3-and 5-year OS rates in the NGGCT poor prognosis group were $42.9 \%$ and $34.3 \%$, respectively.

\section{Toxicity With the Treatment of Intracranial GCTs}

Grade 3 or 4 toxicities were observed in 47 patients $(42.7 \%)$ registered in this study (Table 3 ). No treatmentrelated deaths were observed. Parinaud syndrome, impaired visual acuity, visual field defect, and diplopia were observed in $10.9 \%, 9.1 \%, 8.1 \%$, and $7.3 \%$ of the patients, respectively. Considered as less frequent events, therapyrelated myelodysplastic syndrome was observed in one patient $(0.9 \%)$, and quasi-moyamoya disease was observed in another patient $(0.9 \%)$.

\section{Neurocognitive Outcomes of Long-Term Survivors With Intracranial GCTs}

As of January 1, 2020, 88 patients with intracranial GCTs were alive. Of these, 35 survivors could be contacted by telephone, and they agreed to participate in the TICS-J survey. The mean cognitive score for the TICS-J was $33.5 \pm 5.8$ points $( \pm \mathrm{SD}$; range $8-40)$. 


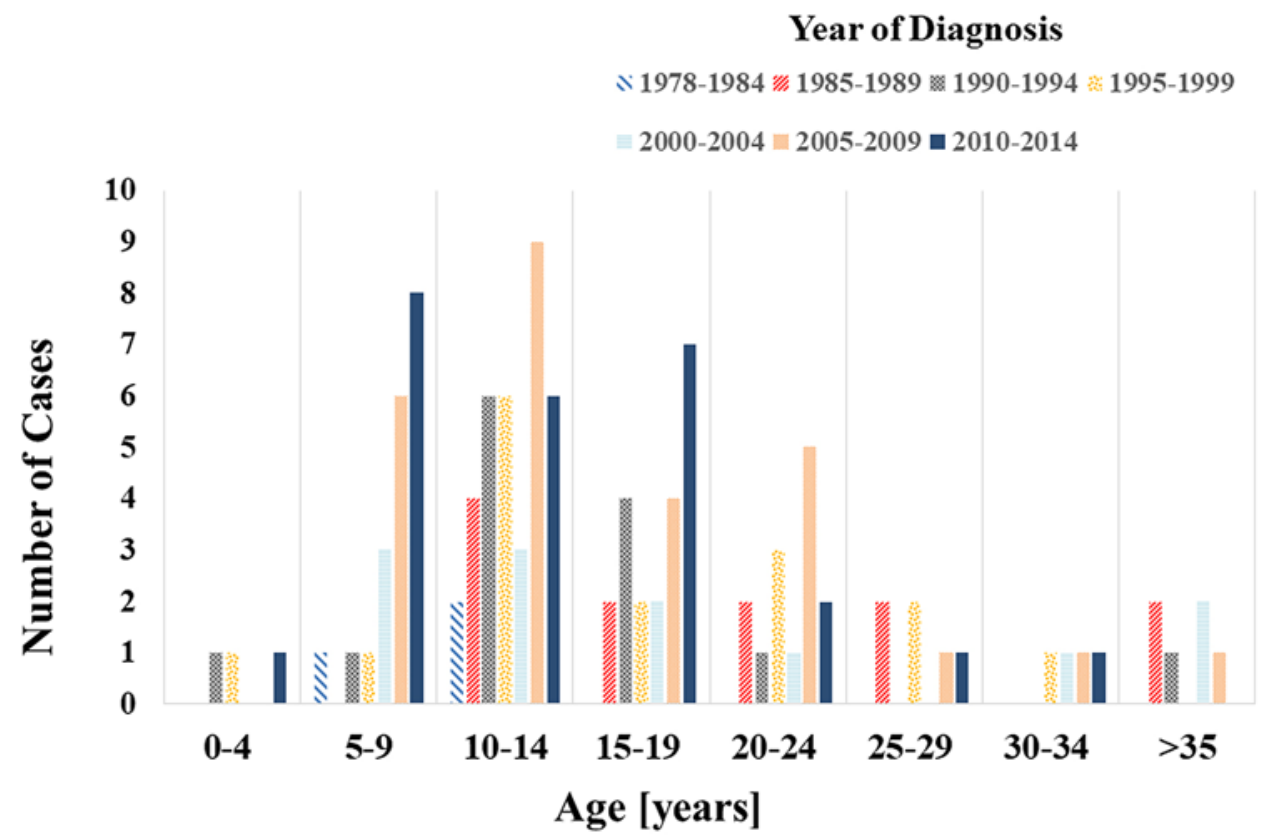

FIG. 1. Age distribution of primary intracranial GCTs at the Nagoya University Hospital by 5 -year calendar period of diagnosis, 1978-2014. Figure is available in color online only.

\section{Univariate and Multivariate Analyses for the Clinical Outcomes of the NGGCT Intermediate Prognosis and Poor Prognosis Groups}

Univariate analysis showed that the OS in the NGGCT intermediate prognosis and poor prognosis groups was significantly associated with age at diagnosis $(\leq 20$ years vs > 20 years: HR 3.43, 95\% CI 1.20-9.77; $p=0.02$ ), AFP levels $(<300 \mathrm{ng} / \mathrm{mL}$ vs $\geq 300 \mathrm{ng} / \mathrm{mL}$ : HR $3.63,95 \%$ CI $1.52-12.83 ; \mathrm{p}=0.006$ ), and tumor resection (GTR vs nonGTR: HR 0.34, 95\% CI 0.12-0.97; p = 0.04) (Table 4).

We further established the multivariate survival models for OS in the NGGCT intermediate prognosis and poor

TABLE 2. Summary of the treatment for intracranial GCTs

\begin{tabular}{|c|c|c|c|c|}
\hline \multirow[b]{2}{*}{ Variable } & \multirow{2}{*}{$\begin{array}{c}\text { Germinomatous } \\
\text { Group }\end{array}$} & \multicolumn{3}{|c|}{ NGGCT Group } \\
\hline & & Mature Teratoma & NGGCT (intermediate prognosis) & NGGCT (poor prognosis \\
\hline No. of patients & 70 & 7 & 19 & 14 \\
\hline \multicolumn{5}{|l|}{ Surgery } \\
\hline Total & 1 & 2 & 12 & 8 \\
\hline Subtotal & 2 & 2 & 6 & 1 \\
\hline Partial & 10 & 3 & 1 & 3 \\
\hline Biopsy & 17 & 0 & 0 & 2 \\
\hline None & 40 & 0 & 0 & 0 \\
\hline \multicolumn{5}{|l|}{ Chemotherapy } \\
\hline CBDCA+VP-16 & 47 & 0 & 10 & 1 \\
\hline CDDP+VP-16 & 15 & 0 & 5 & 2 \\
\hline ICE & 6 & 0 & 3 & 11 \\
\hline None & 2 & 7 & 1 & 0 \\
\hline \multicolumn{5}{|l|}{ Radiotherapy } \\
\hline Whole ventricle & 21 & 0 & 6 & 4 \\
\hline Whole brain & 18 & 0 & 5 & 1 \\
\hline Craniospinal axis & 12 & 0 & 2 & 5 \\
\hline Large focal & 16 & 0 & 4 & 4 \\
\hline None & 3 & 7 & 2 & 0 \\
\hline
\end{tabular}



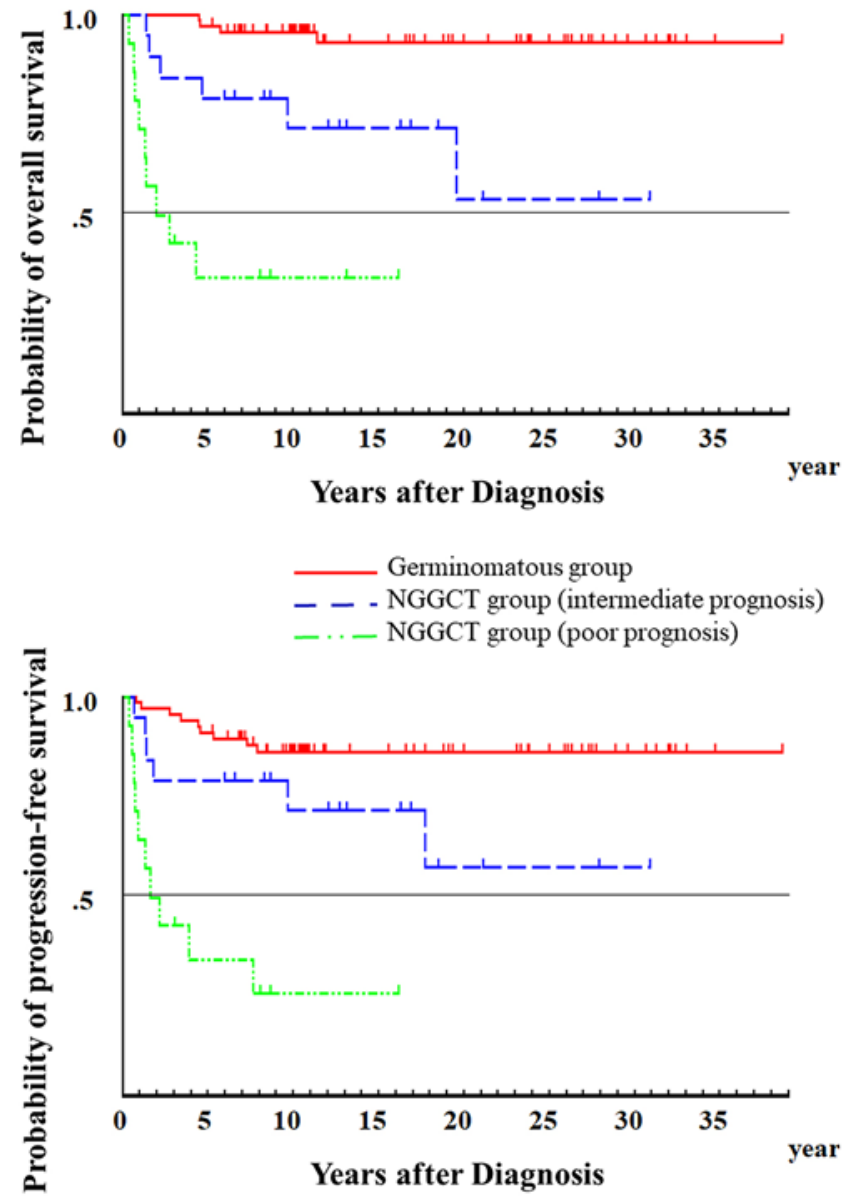

FIG. 2. Upper: Kaplan-Meier curves showing the OS rate of patients in the germinomatous, NGGCT intermediate prognosis, and NGGCT poor prognosis groups. Lower: Kaplan-Meier curves showing the PFS of patients in the germinomatous, NGGCT intermediate prognosis, and NGGCT poor prognosis groups. Figure is available in color online only.

prognosis groups using the following prognostic factors: age at diagnosis, AFP levels, and extent of tumor resection. These multivariate models were subsequently adjusted to estimate the hazard ratio (HR) associated with the effect of OS in these groups. Among these three predictors investigated, only the AFP status was significantly associated with worsened OS (HR 3.88, 95\% CI 1.29-11.66; $\mathrm{p}=$ 0.02) (Table 4).

\section{Discussion}

GCTs are rare, heterogeneous diseases that have been subjected to therapeutic investigation. Given that GCTs are rare tumors, it is considered difficult to conduct largescale prospective studies assessing these types of tumors. Previous reports describing the clinical outcomes of patients with intracranial GCTs are therefore limited, considering their small sample size, selection bias, and variable treatment options. ${ }^{7,15,17,21}$

Germinomas are curable with radiation therapy alone or in combination with neoadjuvant chemotherapy and radiation therapy. One study reported the clinical outcomes
TABLE 3. Adverse events observed during chemoradiotherapy in the intracranial GCTs

\begin{tabular}{lc}
\hline & No. of Patients (\%) \\
\hline Myelosuppression (grade 3 or 4) & $47(42.7)$ \\
\hline Parinaud syndrome & $12(10.9)$ \\
\hline Impaired visual acuity & $10(9.1)$ \\
\hline Visual field defect & $9(8.1)$ \\
\hline Diplopia & $8(7.3)$ \\
\hline Therapy-related myelodysplastic syndrome & $1(0.9)$ \\
\hline Quasi-moyamoya disease & $1(0.9)$ \\
\hline
\end{tabular}

of 24 patients with intracranial germinomas treated with reduced radiation and some chemotherapy agents, including carboplatin, etoposide, and ifosfamide. This study showed that the 5-year PFS and OS rates were $96 \%$ and $100 \%$, respectively. ${ }^{17}$ Another study revealed the promising results of intracranial GCTs that were treated with surgery followed by chemotherapy with etoposide and cisplatin or ifosfamide, cisplatin, and etoposide and lowdose involved-field radiotherapy. ${ }^{10}$ The 5-year OS and PFS rates for patients with pure germinoma were $100 \%$ and $86 \%$, respectively. Furthermore, treatment of germinomas has developed from high-dose craniospinal irradiation to platinum-based chemotherapy followed by radiation therapy to the whole ventricular system because most germinomas relapse within the ventricle. ${ }^{6}$ The current main protocol, the CBDCA+VP-16 regimen followed by radiation therapy $(30 \mathrm{~Gy})$ to the whole ventricle or radiotherapy ( $30 \mathrm{~Gy}$ ) to the whole brain, was used for the patients in the germinomatous group. The present study demonstrated a more favorable outcome of patients in the germinomatous group than outcomes in previous studies, ${ }^{12,22}$ with the 5-, $10-$, and 20 -year OS rates being $97.1 \%, 95.7 \%$, and $93.2 \%$, respectively, and the 5-, 10-, and 20-year PFS rates being $91.4 \%, 86.6 \%$, and $86.6 \%$, respectively. Moreover, in further studies, it would be possible to reduce the radiation doses from 30 Gy to less than 24 Gy, significantly strengthening the preradiation chemotherapy applied to germinomas.

The prognosis of NGGCTs is worse than that of pure germinomas. Because treatment of these tumors was most often radiotherapy following biopsy or partial resection in the 1970s, long-term disease-free survival was only approximately $10 \% .{ }^{23}$ Intensified multimodality treatment, including tumor resection, intensive chemotherapy, and craniospinal irradiation, enhanced the clinical outcomes of patients with NGGCTs. Some recent studies have demonstrated more favorable outcomes of NGGCTs compared with germinomatous tumors. One study evaluating the long-term outcomes of 94 NGGCTs showed that the OS rate for both 20- and 30-year time points for NGGCTs was $86.7 \% .{ }^{14}$ In that study, radiotherapy was the standard initial treatment in $62.8 \%$ of patients with NGGCTs and was combined with surgery in $48.9 \%$ of patients. In the intermediate-risk group, a total tumor-free rate of $55.6 \%$ was achieved. However, for poor-risk patients, treatment with ifosfamide/cisplatin/etoposide chemotherapy followed by $30-$ Gy craniospinal radiotherapy and a $30-G y$ tumor 
TABLE 4. Univariate and multivariate analyses of OS in the NGGCT intermediate prognosis and poor prognosis groups

\begin{tabular}{|c|c|c|c|c|c|c|}
\hline \multirow[b]{2}{*}{ Variable } & \multicolumn{3}{|c|}{ Univariate Analysis } & \multicolumn{3}{|c|}{ Multivariate Analysis } \\
\hline & $\mathrm{HR}$ & $95 \% \mathrm{Cl}$ & $p$ Value & $\mathrm{HR}$ & $95 \% \mathrm{Cl}$ & $p$ Value \\
\hline \multicolumn{7}{|l|}{ Age, yrs } \\
\hline$\leq 20$ & 1 & & & 1 & & \\
\hline$>20$ & 3.43 & $1.20-9.77$ & 0.02 & 2.17 & $0.7-6.69$ & 0.18 \\
\hline \multicolumn{7}{|l|}{ Sex } \\
\hline Female & 1 & & & & & \\
\hline Male & 2.22 & $0.29-16.97$ & 0.44 & & & \\
\hline \multicolumn{7}{|l|}{ Tumor location } \\
\hline Other regions & 1 & & & & & \\
\hline Pineal regions & 1.63 & $0.56-4.78$ & 0.37 & & & \\
\hline \multicolumn{7}{|l|}{ AFP (ng/mL) } \\
\hline$<300$ & 1 & & & 1 & & \\
\hline$\geq 300$ & 3.63 & $1.52-12.83$ & 0.006 & 3.88 & $1.29-11.66$ & 0.02 \\
\hline \multicolumn{7}{|l|}{$\beta-h C G$ (IU/L) } \\
\hline$<300$ & 1 & & & & & \\
\hline$\geq 300$ & 0.87 & $0.20-3.90$ & 0.86 & & & \\
\hline \multicolumn{7}{|l|}{ Tumor resection } \\
\hline Non-GTR & 1 & & & 1 & & \\
\hline GTR & 0.34 & $0.12-0.97$ & 0.04 & 0.45 & $0.154-1.37$ & 0.16 \\
\hline \multicolumn{7}{|l|}{ Multiplicity } \\
\hline- & 1 & & & & & \\
\hline+ & 0.47 & $0.06-3.61$ & 0.47 & & & \\
\hline \multicolumn{7}{|l|}{ Dissemination } \\
\hline- & 1 & & & & & \\
\hline+ & 2.36 & $0.66-8.45$ & 0.19 & & & \\
\hline
\end{tabular}

- = absent; + = present.

Boldface type indicates statistical significance.

boost followed by further adjuvant chemotherapy could not control the poor prognosis for patients with NGGCTs. ${ }^{9}$ According to the European SIOP-CNS-GCT-96 trial, 116 patients with localized malignant NGGCTs had 5-year OS and PFS rates of $82 \%$ and $72 \%$, respectively. ${ }^{24}$ That trial used cisplatin-based chemotherapy followed by radiotherapy (54 Gy focal for localized disease, 30 Gy craniospinal plus a 24-Gy tumor boost to the primary and metastatic sites). Evaluating the effects of neoadjuvant chemotherapy with or without second-look surgery before craniospinal irradiation on the outcomes in children with newly diagnosed NGGCTs, according to the ACNS0122 trial, 102 NGGCT patients had 5-year event-free survival and OS rates of $93 \%$ and $84 \%$, respectively. ${ }^{25}$ Four patients underwent second-look surgery prior to receiving radiation therapy in our series. Two of the 4 patients had residual NGGCTs and immediately died after the treatment (one yolk sac tumor and one mixed tumor mainly comprising a yolk sac tumor). Two patients who had teratomas (one mature teratoma and one immature teratoma) were alive without relapse.

Contrary to these previous studies, $, 14,24,25$ the patients in the NGGCT group in the present study had a relatively unfavorable outcome, with 5-year OS and PFS rates of $67.3 \%$ and $67.4 \%$, respectively. While our study also confirmed the relevance of the classification that divides NGGCTs into three groups, in particular, the 5-year OS rate of the NGGCT poor prognosis group was severely worse, which demonstrated that the 3- and 5-year OS rates in the NGGCT poor prognosis group were $42.9 \%$ and $34.3 \%$, respectively, probably because NGGCT is a significantly heterogeneous group of diseases with different prognoses. This heterogeneity makes the comparison of clinical outcomes from other studies, patient cohorts, and clinical trials difficult. Consequently, treatments for NGGCTs vary, with no consensus on their optimal management. Although the clinical outcomes were still unclear in the NGGCT poor prognosis group in the current study, a new protocol that increases the survival rate of patients in the NGGCT poor prognosis group should be considered.

With an increasing survival rate of patients with GCTs, improving the quality of life by reducing the rate of longterm adverse events induced by the treatment for GCT patients is greatly required. Although Packer et al. reported that a radiation dose of $30 \mathrm{~Gy}$ or greater to any cortex of the brain was associated with a twofold increased risk of late seizure disorders in children with brain tumors, ${ }^{26}$ cases of late seizure were not observed in the present study. Furthermore, other groups reported that the patients re- 
ceived intensive chemotherapy followed by reduced-dose (24 Gy) and reduced-field irradiation for pure germinoma. However, late adverse effects, including endocrine disorder, involuntary movements, ear and labyrinth disorders, seizure, and memory impairment, were observed.$^{27}$ In the present study, all patients-except those with mature teratoma-were treated with intensive chemotherapy followed by radiation therapy ( $30 \mathrm{~Gy}$ to the whole ventricle or to the whole brain or $30 \mathrm{~Gy}$ to the whole brain and spine). However, neurocognitive disorders were not observed in any of the patients in this study. Parinaud syndrome, impaired visual acuity, visual field defect, and diplopia were associated with salvage surgery to the residual tumor after chemotherapy. In our study, 47 of the 110 patients (42.7\%) experienced adverse events involving grade 3 or grade 4 hematological toxicities (Table 3). The incidence of toxicities in this study was lower than those reported in other studies of GCT patients..$^{13,24,28}$ Although these adverse events should be carefully monitored in further trials of chemotherapy for GCTs, all were found to be tolerable in the present study population. No treatment-related deaths were observed. Therefore, treatment-specific severe morbidity, including surgery and chemoradiotherapy, was not observed in this study.

From the data on pineal gland tumors in the SEER (Surveillance, Epidemiology, and End Results) database, poor prognosis was significantly associated with female sex, age older than 18 years, NGGCT, and lack of radiation therapy in treatment. ${ }^{29}$ The SIOP-CNS-GCT-96 trial demonstrated the following significant prognostic indicators: diagnostic AFP level and residual disease after second-look surgery in patients with intracranial NGGCTs. Consistent with these studies, AFP status was significantly associated with worsened OS in the multivariate survival models of the NGGCT intermediate prognosis and poor prognosis groups in the present study. Particularly, 8 of the 14 patients $(57.1 \%)$ in the poor prognosis group had diagnostic serum AFP levels $\geq 300 \mathrm{ng} / \mathrm{mL}$ in the NGGCT poor prognosis group, and 6 of these 8 patients (75\%) with high AFP status died. On the contrary, $\beta$-hCG levels were not associated with poor prognosis. This finding may reflect the fact that the high $\beta$-hCG level may have included some $\beta$-hCG-secreting germinomas, that is, germinoma with STGC.

Recent advancements in our biological understanding can help to establish clinical management decisions for intracranial GCTs in the future through the identification of molecular targets. These targets will help in determining the selection of novel therapeutic agents particularly for the NGGCT patient population. According to a previous study from the Intracranial Germ Cell Tumor Genome Analysis Consortium (iGCT Consortium) on genomic features of intracranial GCTs, KIT mutations were observed in $40.0 \%$ of pure germinomas and $5.7 \%$ of NGGCTs. ${ }^{30}$ In contrast, RAS mutations were observed in $20.0 \%$ of pure germinoma and $2.9 \%$ of NGGCTs. Most NGGCT cases with KIT or $R A S$ mutations were actually mixed GCTs including a germinoma component. Therefore, different from pure germinoma, it was indicated that choriocarcinomas, yolk sac tumors, and embryonal carcinomas in the NGGCT poor prognosis group are less dependent on KIT/RAS signaling.
Another study from the iGCT Consortium reported a combined genomic and epigenomic study in the series of intracranial GCTs. ${ }^{31}$ Although microdissected germinoma and NGGCT components of mixed GCTs possess the common KIT/RAS mutations, it was demonstrated that they had distinct global DNA methylation profiles, which show hypomethylation in germinoma components and hypermethylation in NGGCT components. Therefore, these findings suggest that mixed GCTs may develop from the same cell of origin with KIT/RAS mutation. Thereafter, NGGCT components can be derived from germinomas through epigenetic reprogramming. These findings can develop the understanding of the complex mechanisms of intracranial GCT development and will help in establishing different treatment strategies depending on clinical and molecular factors in patients with intracranial GCTs.

Although the present study provides novel information about the long-term survival in patients with primary intracranial GCTs, this study has several limitations. First, our results are more limited than the results of the prospective clinical trials, considering that retrospective studies may be influenced by unrecognized bias. Second, there was no central review to confirm the eligibility for this study inclusion based on appropriate pathological diagnosis. Third, there might be missing data about the information of intracranial GCTs because of the significantly long follow-up period in this study; the median follow-up time was 11.0 years. Finally, the present study comprised a small sample size; specifically, few patients were included in the NGGCT intermediate prognosis and poor prognosis groups in this study.

\section{Conclusions}

Although this was a retrospective analysis, our study showed that platinum-based chemotherapy and radiotherapy result in favorable survival outcomes in patients with the germinomatous type of GCTs. The germinomatous group had a favorable 20 -year OS rate of over $90 \%$. Moreover, the high OS rates were accompanied by a good objective neurological outcome. Clinical outcomes were still unfavorable in the NGGCT intermediate prognosis and poor prognosis groups; however, a new protocol that increases the survival rate of patients belonging to both groups should be considered.

\section{Acknowledgments}

This work was supported by a Grant-in-Aid for Scientific Research (C) to K.M. (no. 17K10862) from the Japan Society for the Promotion of Science (JSPS).

\section{References}

1. Brain Tumor Registry of Japan (2005-2008). Neurol Med Chir (Tokyo). 2017;57(1 Suppl):9-102.

2. Ostrom QT, Cioffi G, Gittleman H, et al. CBTRUS statistical report: primary brain and other central nervous system tumors diagnosed in the United States in 2012-2016. Neuro Oncol. 2019;21(suppl 5):v1-v100.

3. McCarthy BJ, Shibui S, Kayama T, et al. Primary CNS germ cell tumors in Japan and the United States: an analysis of 4 tumor registries. Neuro Oncol. 2012;14(9):1194-1200.

4. Louis DN, Ohgaki H, Wiestler OD, et al. The 2007 WHO 
classification of tumours of the central nervous system. Acta Neuropathol. 2007;114(2):97-109.

5. Louis DN, Perry A, Reifenberger G, et al. The 2016 World Health Organization Classification of Tumors of the Central Nervous System: a summary. Acta Neuropathol. 2016;131(6): 803-820.

6. Murray MJ, Bartels U, Nishikawa R, et al. Consensus on the management of intracranial germ-cell tumours. Lancet Oncol. 2015;16(9):e470-e477.

7. Dufour C, Guerrini-Rousseau L, Grill J. Central nervous system germ cell tumors: an update. Curr Opin Oncol. 2014; 26(6):622-626.

8. Kong Z, Wang Y, Dai C, et al. Central nervous system germ cell tumors: a review of the literature. J Child Neurol. 2018; 33(9):610-620.

9. Matsutani M. Combined chemotherapy and radiation therapy for CNS germ cell tumors-the Japanese experience. J Neurooncol. 2001;54(3):311-316.

10. Aoyama H, Shirato H, Ikeda J, et al. Induction chemotherapy followed by low-dose involved-field radiotherapy for intracranial germ cell tumors. J Clin Oncol. 2002;20(3):857-865.

11. Bouffet E, Baranzelli MC, Patte C, et al. Combined treatment modality for intracranial germinomas: results of a multicentre SFOP experience. Société Française d'Oncologie Pédiatrique. Br J Cancer. 1999;79(7-8):1199-1204.

12. Matsutani M, Sano K, Takakura K, et al. Primary intracranial germ cell tumors: a clinical analysis of 153 histologically verified cases. J Neurosurg. 1997;86(3):446-455.

13. Calaminus G, Kortmann R, Worch J, et al. SIOP CNS GCT 96: final report of outcome of a prospective, multinational nonrandomized trial for children and adults with intracranial germinoma, comparing craniospinal irradiation alone with chemotherapy followed by focal primary site irradiation for patients with localized disease. Neuro Oncol. 2013;15(6): 788-796.

14. Acharya S, DeWees T, Shinohara ET, Perkins SM. Long-term outcomes and late effects for childhood and young adulthood intracranial germinomas. Neuro Oncol. 2015;17(5):741-746.

15. Foo AS, Lim C, Chong DQ, et al. Primary intracranial germ cell tumours: experience of a single South-East Asian institution. J Clin Neurosci. 2014;21(10):1761-1766.

16. Kochi M, Itoyama Y, Shiraishi S, et al. Successful treatment of intracranial nongerminomatous malignant germ cell tumors by administering neoadjuvant chemotherapy and radiotherapy before excision of residual tumors. J Neurosurg. 2003;99(1):106-114.

17. Cheng S, Kilday JP, Laperriere N, et al. Outcomes of children with central nervous system germinoma treated with multi-agent chemotherapy followed by reduced radiation. $J$ Neurooncol. 2016;127(1):173-180.

18. Kanamori M, Kumabe T, Saito R, et al. Optimal treatment strategy for intracranial germ cell tumors: a single institution analysis. J Neurosurg Pediatr. 2009;4(6):506-514.

19. Yoshida J, Sugita K, Kobayashi T, et al. Prognosis of intracranial germ cell tumours: effectiveness of chemotherapy with cisplatin and etoposide (CDDP and VP-16). Acta Neurochir (Wien). 1993;120(3-4):111-117.

20. Konagaya Y, Washimi Y, Hattori H, et al. Validation of the telephone interview for cognitive status (TICS) in Japanese. Int J Geriatr Psychiatry. 2007;22(7):695-700.

21. Fetcko K, Dey M. Primary central nervous system germ cell tumors: a review and update. Med Res Arch. 2018;6(3):1719.
22. Kawabata Y, Takahashi JA, Arakawa Y, et al. Long term outcomes in patients with intracranial germinomas: a single institution experience of irradiation with or without chemotherapy. J Neurooncol. 2008;88(2):161-167.

23. Jennings MT, Gelman R, Hochberg F. Intracranial germ-cell tumors: natural history and pathogenesis. J Neurosurg. 1985; 63(2):155-167.

24. Calaminus G, Frappaz D, Kortmann RD, et al. Outcome of patients with intracranial non-germinomatous germ cell tumors-lessons from the SIOP-CNS-GCT-96 trial. Neuro Oncol. 2017;19(12):1661-1672.

25. Goldman S, Bouffet E, Fisher PG, et al. Phase II trial assessing the ability of neoadjuvant chemotherapy with or without second-look surgery to eliminate measurable disease for nongerminomatous germ cell tumors: a Children's Oncology Group study. J Clin Oncol. 2015;33(22):2464-2471.

26. Packer RJ, Gurney JG, Punyko JA, et al. Long-term neurologic and neurosensory sequelae in adult survivors of a childhood brain tumor: childhood cancer survivor study. J Clin Oncol. 2003;21(17):3255-3261.

27. Takada A, Ii N, Hirayama M, et al. Long-term follow-up of intensive chemotherapy followed by reduced-dose and reduced-field irradiation for intracranial germ cell tumor. $J$ Neurosurg Pediatr. 2018;23(3):317-324.

28. Lee DS, Lim DH, Kim IH, et al. Upfront chemotherapy followed by response adaptive radiotherapy for intracranial germinoma: prospective multicenter cohort study. Radiother Oncol. 2019;138:180-186.

29. Al-Hussaini M, Sultan I, Abuirmileh N, et al. Pineal gland tumors: experience from the SEER database. J Neurooncol. 2009:94(3):351-358.

30. Fukushima S, Otsuka A, Suzuki T, et al. Mutually exclusive mutations of KIT and RAS are associated with KIT mRNA expression and chromosomal instability in primary intracranial pure germinomas. Acta Neuropathol. 2014;127(6): 911-925.

31. Fukushima S, Yamashita S, Kobayashi H, et al. Genomewide methylation profiles in primary intracranial germ cell tumors indicate a primordial germ cell origin for germinomas. Acta Neuropathol. 2017;133(3):445-462.

\section{Disclosures}

The authors report no conflict of interest concerning the materials or methods used in this study or the findings specified in this paper.

\section{Author Contributions}

Conception and design: Motomura, Yoshida, Natsume, Wakabayashi. Acquisition of data: Motomura, Shimizu, Ohka, Aoki, Tanahashi, Hirano, Chalise, Nishikawa, Yamaguchi. Analysis and interpretation of data: Motomura, Shimizu. Drafting the article: Motomura, Shimizu. Statistical analysis: Aoki. Study supervision: Yoshida, Natsume, Wakabayashi.

\section{Correspondence}

Kazuya Motomura: Nagoya University School of Medicine, Nagoya, Japan.kmotomura@med.nagoya-u.ac.jp. 